\title{
ACOUSTIC MONITORING OF TERRORIST INTRUSION IN A DRINKING WATER NETWORK
}

\author{
B.A.J. Quesson ${ }^{1}$, M.K Sheldon-Robert ${ }^{1}$, I.N. Vloerbergh ${ }^{2}$, J.H.G Vreeburg ${ }^{2}$ \\ ${ }^{1}$ TNO Defence Security and Safety, underwater technology department \\ The Hague, The Netherlands; email: \{benoit.quesson;myriam.sheldon\}@tno.nl \\ ${ }^{2}$ KIWA Water Research \\ Nieuwegein, The Netherlands; email: \{irene.vloerbergh;jan.vreeburg\}@kiwa.nl
}

\begin{abstract}
In collaboration with Kiwa Water Research, TNO (Netherlands Organisation for Applied Scientific Research) has investigated the possibilities to detect and classify aberrant sounds in water networks, using acoustic sensors. Amongst the sources of such sounds are pumps, drills, mechanical impacts, which could indicate, for instance, a terrorist's attempt to inject a toxicant into the water mains. In parallel, an important spin-off is recognised in the detection of other sounds, such as those caused by leaks in water pipes and leaking valves. The acoustic water pipe monitoring project was carried out between 2003 and 2007 in three distinct phases:

- The first phase was carried out in 2003-2004 to gain knowledge on the subject. Models were developed for the sound propagation in water pipes and computer simulations were carried out to specify detection ranges.

- In the second phase, in 2005-2006, the knowledge created in phase 1 was applied for detection of leakage and terrorist attacks. To this end, a number of field experiments were carried out to characterise background noise and suspicious sounds. It was shown that both leakage and intrusion sounds could be detected up to a range of $100 \mathrm{~m}$ in regular 110-mm diameter PVC pipes. At this point, Kiwa Water Research and TNO decided to focus their efforts on terrorist detection in the third and last phase of the project.

- In the third phase, carried out in 2006-2007, a demonstrator was developed for the detection and classification of aberrant sounds and tested on an operational water supply system.

Overall, the TNO acoustic monitoring demonstrator, equipped with two sensors fixed on fire hydrants and two on service taps, has proven to be a suitable and promising system for protection of buildings: it is capable of working in real time and detected about $90 \%$ of terrorist intrusion sounds within a range of $110 \mathrm{~m}$ (a range suitable for building protection). Using the techniques developed for leakage detection in phase 2, and a similar acoustic demonstrator, the performance of operational systems could further be assessed to detect leakage.
\end{abstract}

\section{INTRODUCTION}

The dependence of cities on their water supplies has been known for centuries, and not so long ago it was common practice for attackers in a siege to throw infected corpses (e.g. with plague) in the besieged city's wells. Today, terrorists' threats to the water distribution infrastructure are taken very seriously. The city of Montreal, for instance, recently started a 4-year renovation and fortification programme of its water systems to prevent vandalism and terrorist attacks (Le Devoir, 5 October 2007).

In the water medium, it is well known that sound waves can propagate quickly: typically with a speed of $1500 \mathrm{~m} / \mathrm{s}$ versus typically $340 \mathrm{~m} / \mathrm{s}$ in the air and over long distances depending on frequency content and 
local conditions. As an example, blue whales can communicate with each other at distances of up to several thousands of kilometres (Hiyoshi, 2004). These properties have already been exploited for a long time in sonar systems. The project's purpose was to ally the expertise of the TNO underwater technology group in this domain to the expertise of KIWA Water Research on water management in order to monitor terrorist intrusions by detecting aberrant sounds. As mentioned before, leakage sounds were also monitored and measured during this project but the focus of this paper will mainly be on detection and classification of terrorist intrusions in the drinkable water network.

In order to be able to design a system for detection and classification of sounds in a water network, a literature study was performed to build up a theoretic base for understanding sound propagation in water pipes and get an idea of the state of the art in this field. In parallel to this theoretical study, a first test measurement was conducted in a partly buried PVC pipe to test and validate the propagation models and to measure a few typical sounds to further develop detection and classification algorithms. Subsequently, three sets of acoustic measurements were carried out in different settings (ranging from fully controlled to semi-controlled settings) to associate recorded sounds with (controlled) noise activities and to relate those to relevant parameters such as distance and background noise.

Because the drinking water infrastructure in the Netherlands is often made from PVC and buried underground it was not possible to detect sounds from distances greater than $100 \mathrm{~m}$. Aberrant sounds within this range could be detected and classified using a monitoring system especially designed for this purpose. Because of the limited range, this system is more suited for building protection.

\section{LITERATURE STUDY AND FIRST TEST}

Examining scientific journals, a patent database and the internet, a considerable amount of useful information was found with respect to acoustic monitoring of pipelines in general (Van Walree, 2004), although no references were found which bracket together acoustic monitoring and terrorist intrusion. The most frequently found application is monitoring to detect leaks in fluid or gas pipelines. Various algorithms are mentioned as candidate leak detectors, whereas the cross correlation technique seems to be the most suitable candidate for localization of an event. It appeared to be difficult to estimate in general source levels and time/frequency characteristics (signatures) of suspicious noises in water pipes.

Because a certain basic knowledge of such sounds is indispensable for the evaluation of signal-to-noise ratios (SNRs) and detection-range predictions, it was decided to perform a "quick-and-dirty" field test. For this purpose a hydrophone was mounted in a standard service tap, and used to record water pipe sounds in an urban environment in the Netherlands (see Figure 1). The test site featured pipes of various diameters, but all made of PVC. A variety of sounds were recorded, such as the opening and closure of valves and fire hydrants, hammer ticks on the pipes inside a nearby apartment building, and the water pumps inside the same building. 

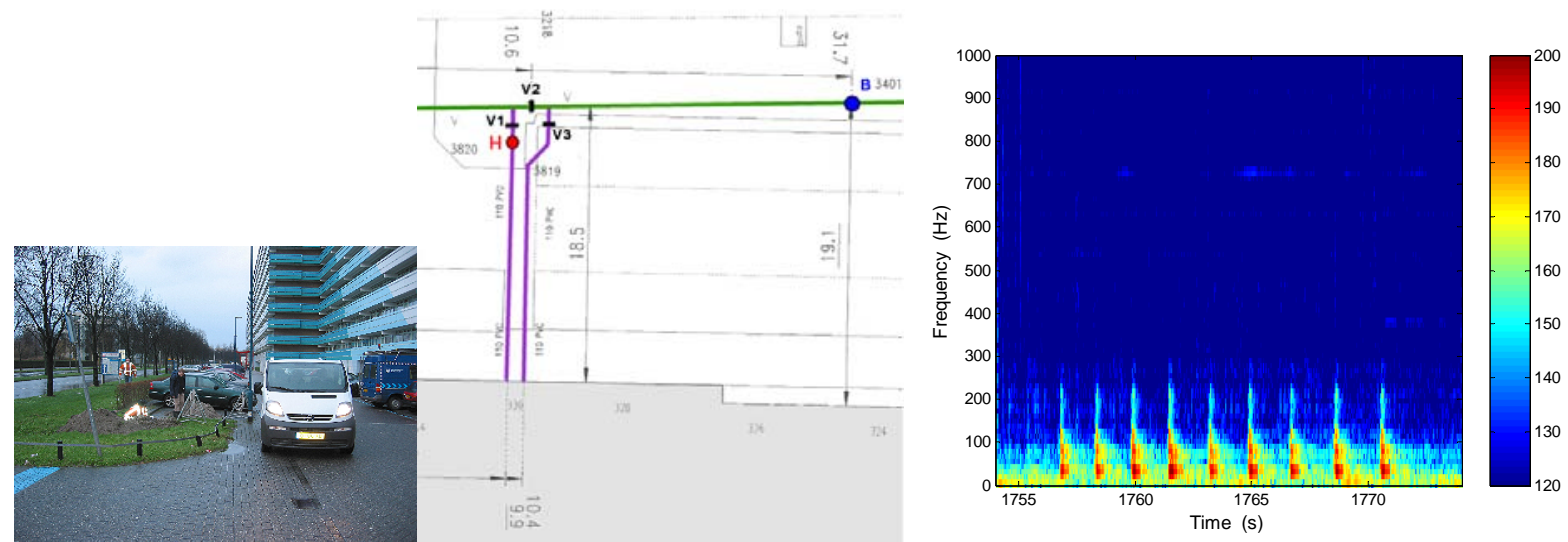

Figure 1 - left hand side: view of the test site. Middle: Sketch of the test site (zoom around the hydrophone). $\mathrm{H}$ = hydrophone; V1 = valve 1; V2 = valve 2; V3 = valve $3, \mathrm{~B}=$ fire hydrant. The light grey area is the apartment building. Distances are in meters. Right hand side: Spectrogram of 9 hammer ticks in the apartment's pump room. Distance from hydrophone: about 25 meters.

The recorded sounds (see an example in Figure 1, right hand side) were subsequently analysed in the TNO laboratory. It was possible to detect the opening of a fire hydrant at a distance of $125 \mathrm{~m}$ (maximum distance tested) from the monitoring station. This hydrant was the furthest source of sound that was tested during the experiments. Furthermore, almost all intentionally made sounds were detected with the Pagetest algorithm. One signal was missed when the pumps inside the building were switched on; the Page test detector was not tuned properly at that time to filter out this pump as a background noise.

\section{Sound propagation in buried water pipes filled with water}

The available literature and models for sound propagation in water pipes (see Van Walree 2004 section 5.3) are limited in application to the frequency range below $1 \mathrm{kHz}$, where the current acoustic leak detection techniques are applied. These models indicate that the attenuation of sound in PVC pipes increases strongly with distance and with frequency, which means that the detection of sounds in the frequency range between $100 \mathrm{~Hz}$ and $1 \mathrm{kHz}$ is limited to relatively short distance (of the order of $100 \mathrm{~m}$ ). This is partly confirmed by the results of the measurements. Literature and model calculations show that the detection ranges in this frequency band will be significantly longer in steel, cast iron and concrete water pipes. A model based on the available literature is proposed and implemented (Van Walree, 2004) for the sound propagation in buried water-filled pipes.

\section{Detection algorithms}

A transient, in the sonar field, refers to an unexpected change in the acoustical environment, typically a non natural signal limited in duration such as a very short hammer bang or relatively longer whale whistles. For the water pipe monitoring, little is known about the typical transients to be expected, only that they are short signals and often have a more or less white spectrum. In such cases the only available detector is an energy detector. Such a detector evaluates the received acoustic energy and by application of a detection threshold it is decided whether or not a signal (excess of energy) is present in the noisy background. If little is known about the signal, it is possible to do better. If for instance the frequency contents of the signal is known filters can be applied. For narrowband or coloured-spectrum signals energy detection in the frequency domain (spectrum) is a good option. It is quite easy to transfer acoustic data to the frequency domain by means of Fast Fourier Transforms (FFTs). The problem with a frequency domain approach is the unknown duration, which makes it hard to find the optimum integration time (= FFT length). A popular compromise between time domain and frequency domain approaches is energy detection in the spectrogram (time-frequency plot), which is a sequence of short-time Fourier transforms 
(STFT). Taking these considerations into account it seems wise to use a spectrogram based detector. With the spectrogram as input, still some alternatives for standard energy detection are available. TNO decided to implement a detector, which utilises the Page test (Page, 1954 and Abraham, 1996) which is an optimum detector for signals with unknown duration.

\section{EXPERIMENTAL SUPPORT}

Assembling the knowledge built in the first phase by means of the extensive literature study and the first recorded sounds, an experimental system was developed and tested. It was intended to support experimentally and on a larger scale, in terms of data collection and test locations, what was found and modelled previously. The experimental system consisted of four sensors: two service tap hydrophones and two hydrophones with a special fire hydrant mount (Figure 2, left hand side). These sensors are coupled with a data acquisition system and linked to a PC where the acoustic data are stored. The system is used for the acoustic monitoring of water pipes in three different locations in the Netherlands: an experimental set-up in a forest near Soestduinen (figure 3), a new-housing estate under construction near Amersfoort (figure 4), and a regularly inhabited district in Helmond (figure 5). This allowed to go from a very well controlled environment to a more realistic one (standing water network). The pipe material was 110-mm PVC in all cases to allow comparisons in the three different conditions. The experiments were aimed at characterisation of noise produced by leaking valves, and at so-called "terrorist intrusion" type of noise sources such as hammer ticks, a drill used to mount a service tap, and a pump. The recorded data from the field trials were used to validate sound propagation models and to develop and test detection algorithms. As mentioned in the introduction, this paper only discusses the terrorist intrusion results.

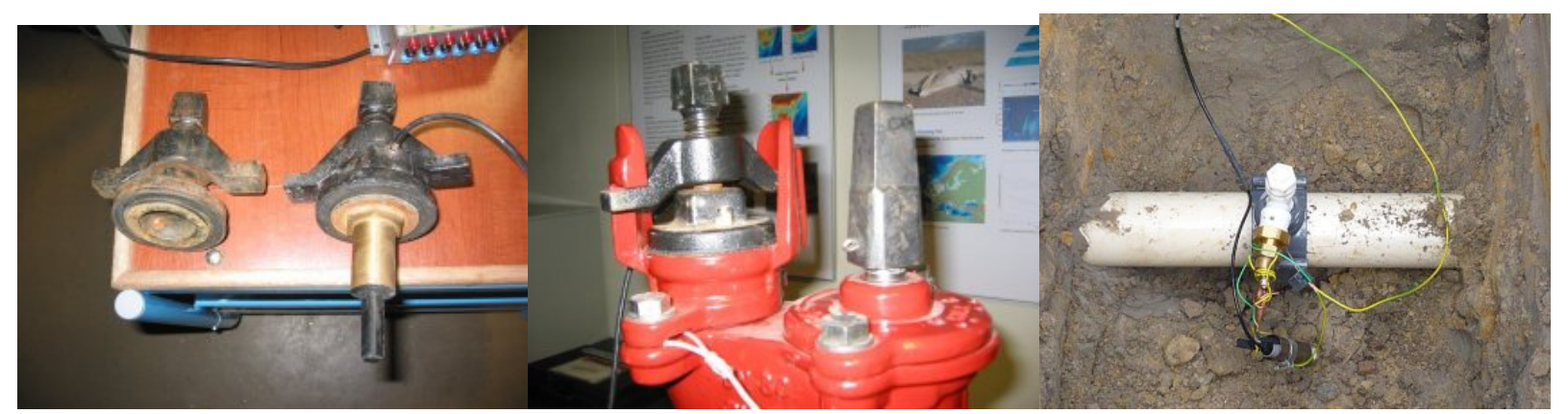

Figure 2 - On the left hand side: one of the two dedicated hydrophones mounted in a specific housing that fits a fire hydrant. Middle: same hydrophone mounted on top of a fire hydrant. On the right hand side, one of the two hydrophones on an installed service tap. The yellow cable for earth is visible and the grey tube at the bottom contains a pre-amplifier. 


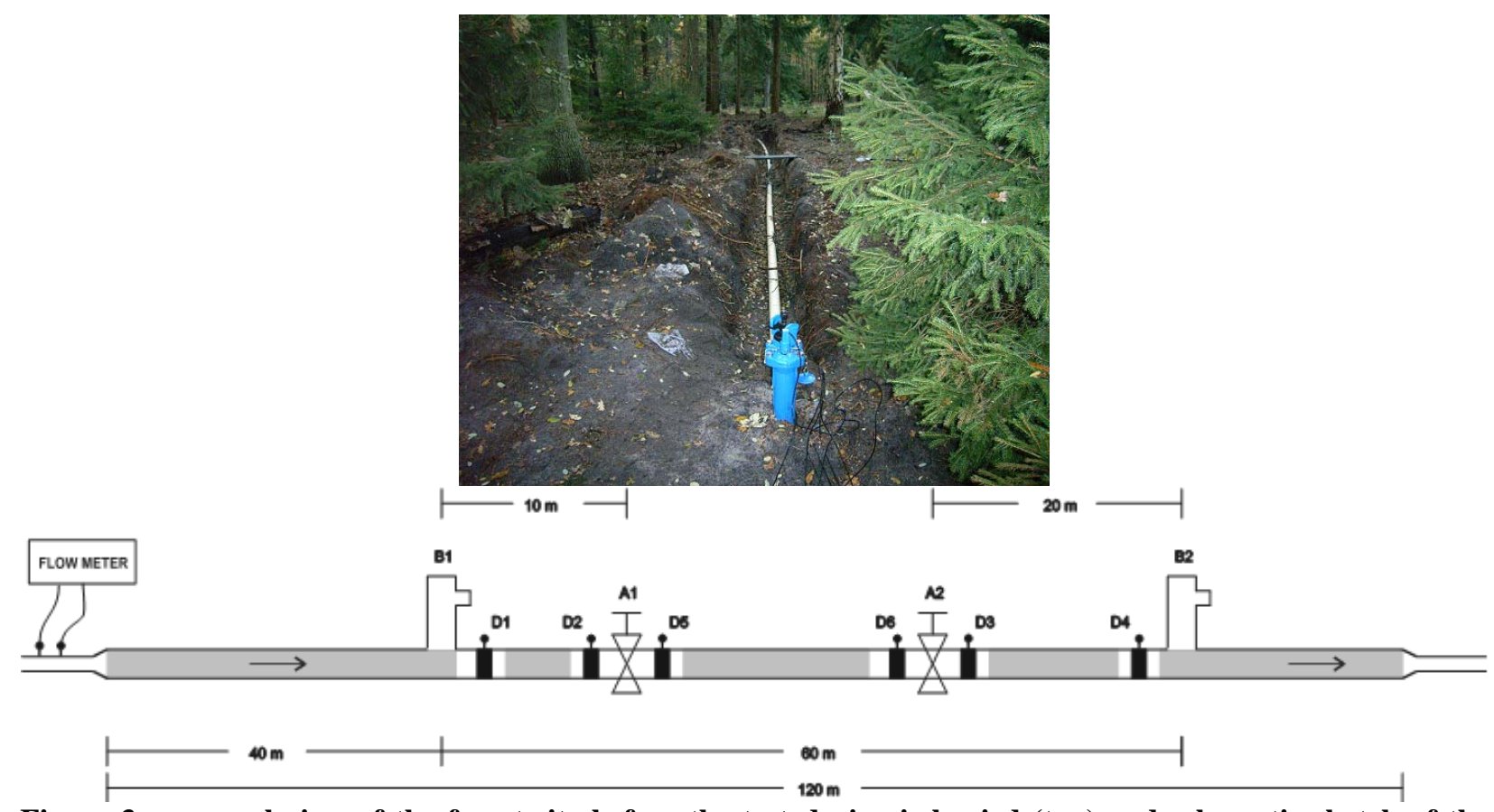

Figure 3 - general view of the forest site before the tested pipe is buried (top) and schematic sketch of the same site (bottom). The grey parts of the pipe are buried. Input and output of water was done with a stiff 63$\mathrm{mm}$ tube with a length of several tens of meters. The experimental pipe itself is a 110-mm PVC pipe. B1 and $B 2$ are two fire hydrants. Dx indicates service tap $x, A 1$ and $A 2$ are two valves.

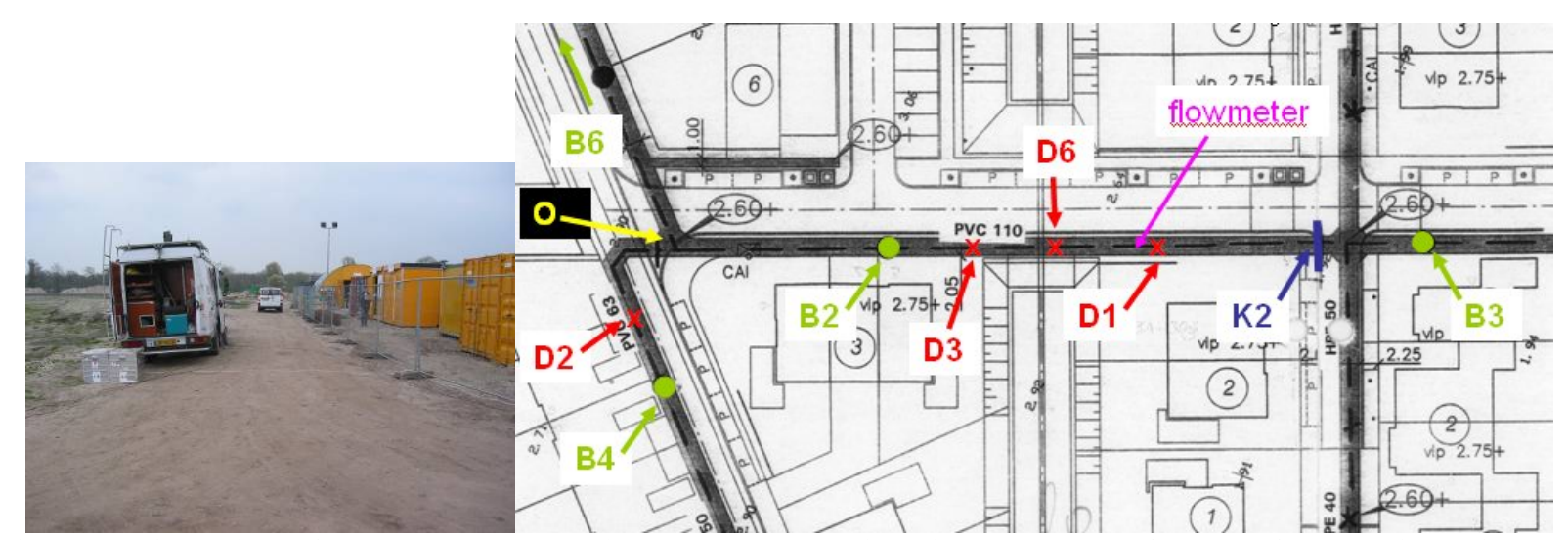

Figure 4 - Overview of measurement location on a construction site near Amersfoort. Left hand side: picture of the site, the measurement van is visible. Right hand side: Map of measurement area: $B x$ indicates fire hydrant $x, \mathrm{D} x$ indicates service tap $x$, en $\mathrm{K} x$ means valve $x$. The distance B2 - B3 is $60.0 \mathrm{~m}$. 


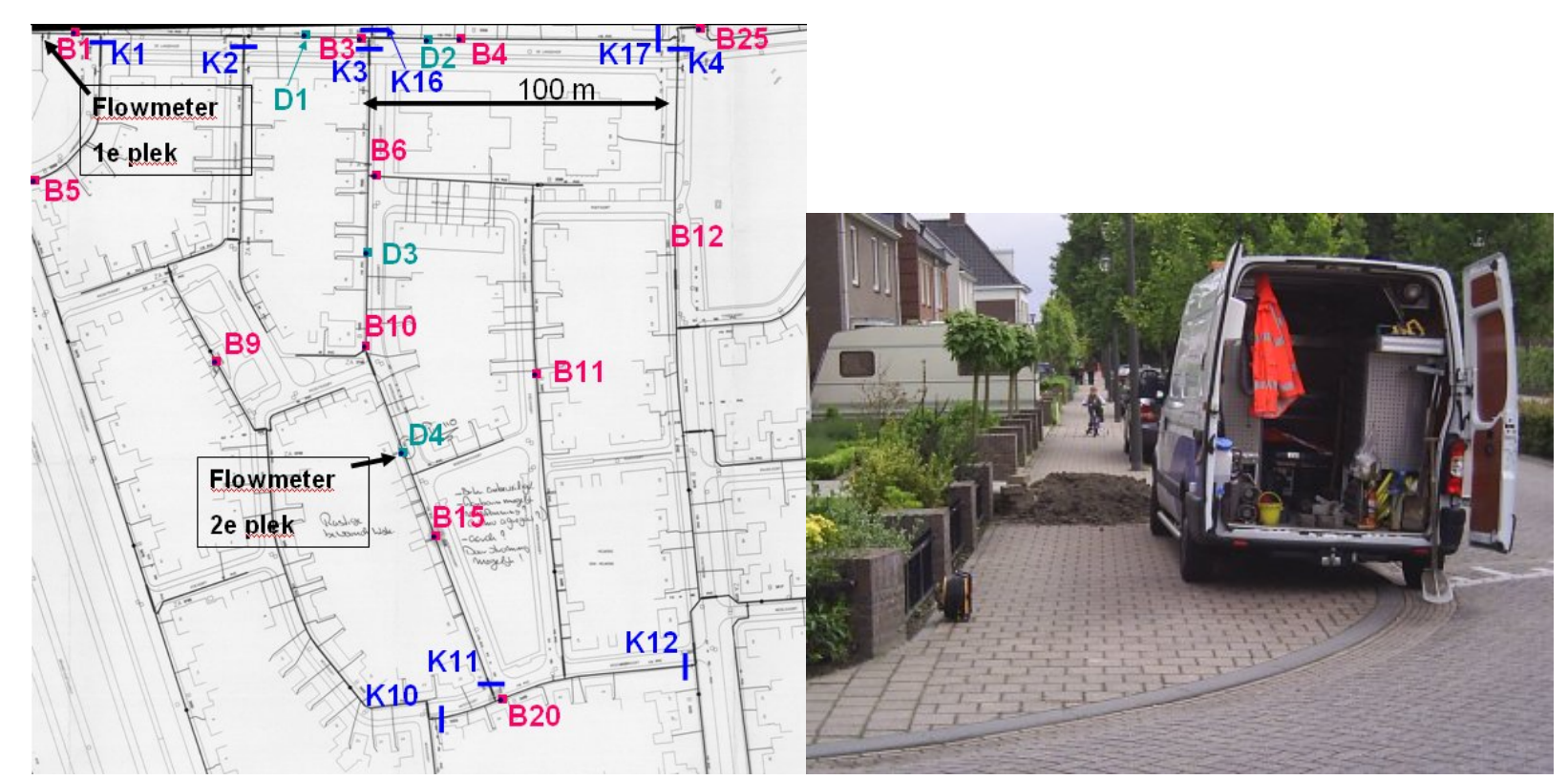

Figure 5 - Overview of measurement site in Helmond. Left hand side: Map of measurement area. $B x$ indicates fire hydrant $x$, Dx indicates service tap $x$, en $\mathrm{K} x$ means valve $x$. On the top right corner a physical distance of $100 \mathrm{~m}$ is indicated. Right hand side: picture of this residential area.

\section{Validation of the propagation model}

In order to validate the propagation model developed previously, the best measurements in terms of quality and control of the external parameters, such as the distance and ambient noise, for this study appeared to be the ones referred to as ID 209 (open pipe) and ID 230 (buried pipe) in the Soestduinen forest (Figure 3, top). These are measurements with "hammer ticks" on the pipe. A time interval was chosen when one would tick with the hammer between positions D1 and D2 (Figure 3, bottom). It was then possible to measure the acoustic propagation between the hydrophones installed on position D5 and D6. This section was chosen because there are no obstructions in the pipe between the two measuring points. The distance between D5 and D6 is $27.75 \mathrm{~m}$.

In Figure 6 (left hand side), the experimental results are presented and compared to the model. This is due partly to the higher damping of sound between D5 and D6, but also to the different characteristics of both hydrophones above this frequency (see Appendix F in De Theije, 2006). These figures show that the model gives a good indication of the actual propagation.

The experiments in Soestduinen enabled the validation of the propagation model, suggested during the preliminary tests, up to $300 \mathrm{~Hz}$. Above $300 \mathrm{~Hz}$ the propagation loss was so strong that the signal-to-noise ratio at the hydrophones was insufficient to quantify the loss. This shows that large-distance detection of events at high frequencies is unlikely. Based on the propagation model and on the source levels, it is also possible to determine the optimal frequency range for the detection method.

\section{Detection of terrorist sounds}

Sounds that are associated with a terrorist attack on a water-supply system have been examined. Hammer ticks on service taps, fire hydrants, and on the pipe itself could be detected on remote hydrophones. Typical detection ranges for Soestduinen are as follows: Roughly half of the hammer ticks at ranges between 7-60 m could be detected with a SNR greater than 4 . This number was chosen as a rough rule- 
of-thumb where one can say that SNRs above 4 can be detected unambiguously. All hammer ticks could be detected within a $7 \mathrm{~m}$ range.

For the Amersfoort experiments it was found that the detection range is different for the flow/no flow situations and also for the upstream or downstream configuration. In general the detection ranges varied between $40-100 \mathrm{~m}$. It is worth mentioning that hammer ticks are uncalibrated, i.e. hammer ticks' strength is not controlled. Therefore a hammer tick detected in one situation could have remained undetected if the tick was a bit weaker.

Only a few recordings are available of mounting a service tap; the used drill could be detected over a range of $50 \mathrm{~m}$ in Soestduinen. A membrane pump was employed in Soestduinen to pump water into the pipe. This pump revealed a pronounced acoustic signature with a very high SNR even after a travel distance of $50 \mathrm{~m}$. Detection ranges for hammer ticks and drills seem restricted to values below $100 \mathrm{~m}$ in PVC.
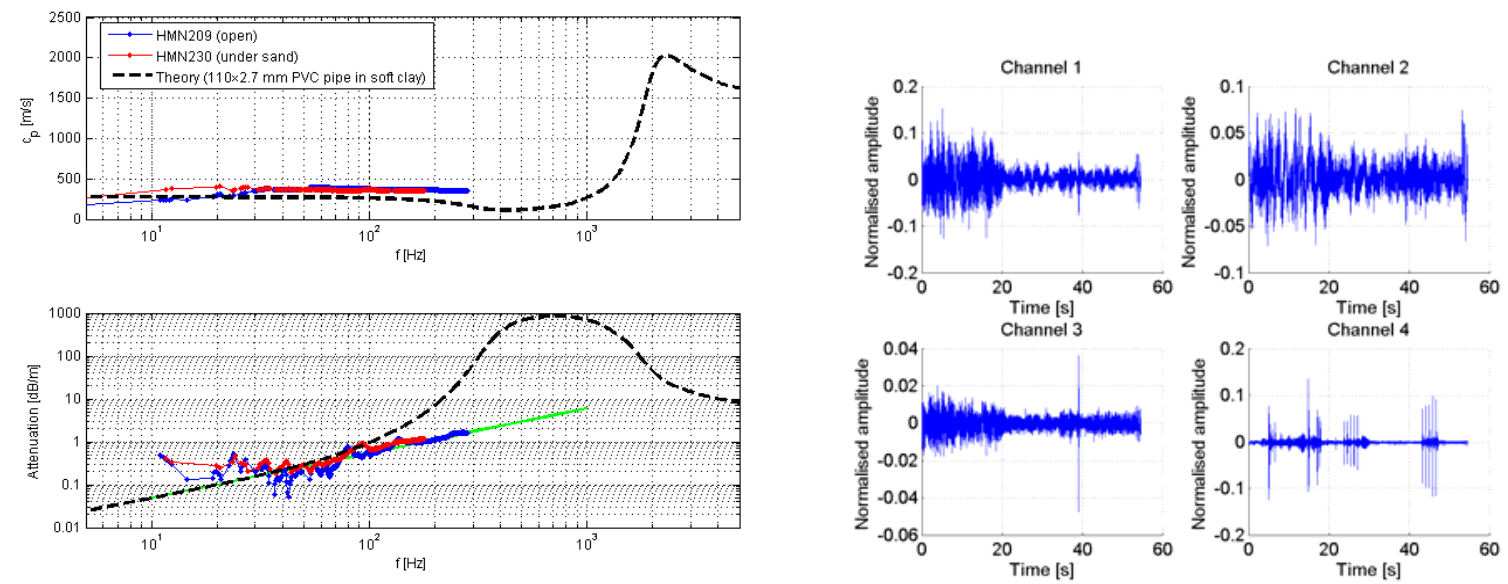

Figure 6 - Left hand side: Comparison between modelled (black, green lines) and measured (blue, red lines) sound velocity (top) and attenuation of sound waves (bottom) in water-filled pipes. Right hand side: Time series recorded during an experiment near Amersfoort (hammer ticking on D4). Channel 1 corresponds to B2, channel 2 to B3, channel 3 to D2 and channel 4 to D2 (see sketch in Figure 4).

\section{PROTECTION OF BUILDINGS}

The experimental support phase made clear that the development of two specific classification algorithms for both leakage sounds and "terrorist sounds" would need too much time. Since long range detection is limited in PVC pipes as demonstrated in the first two phases, it was decided in mutual agreement, that phase 3 would focus only on building protection (Quesson, 2008). The technical aim was twofold: first collect a large database to train and adapt the classification algorithm and then test the real time aspects for the detection. The idea behind is also to install the TNO monitoring system on a fixed location in the same way as it could be used in practice, once a prototype is built. A building was chosen for this purpose offering a controlled and realistic environment (see Figure 7). 


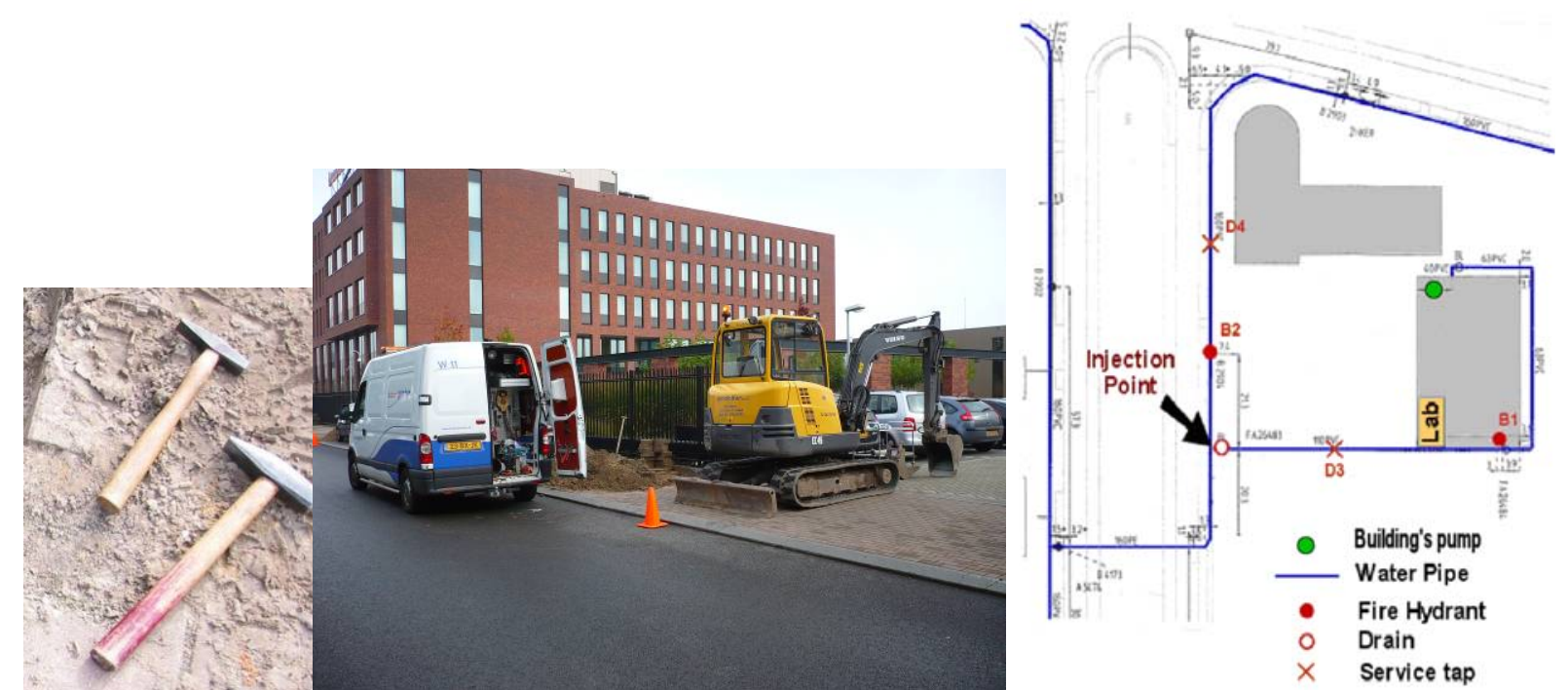

Figure 7 - Left hand side: Two of the three hammers tested as sound source. Middle: View of the selected building, the injection point is located right outside the fence in between the trucks. Right hand side: Sketch of the setup: A terrorist tries to inject, at injection point, a substance in the drinking water pipe (bold blue lines) leading to the building to be protected. The two standing fire hydrants (B1 and B2) and the drain point are represented with a full red circle and empty red circle respectively, the building's pump is represented by a full green circle. The two extra service taps (D3 and D4) installed for the experiment are represented by red crosses.

A large variety of sounds are produced and tested on the injection point to fit the terrorist scenario, also a few of them are tested on the different hydrophones' locations (Figure 7, right hand side). Different sounds are tested, the two main types being hammer ticks and pumps. Hammer ticks are of different strength and produced with three different hammers: a rubber hammer, a big metal hammer and a small metal hammer (these last two are visible in Figure 7, left hand side). Three different pumps are tested (two electrical ones and a manual one) and only from the injection point. Some extra ambient noise test are done such as (controlled) passing cars or trucks, closing and opening of the electric gate (visible behind the trucks in the middle picture in Figure 7). Other sound opportunities were recorded during material installation such as hammering, drilling, digging, mounting and human conversations next to the injection point.

\section{Real time detection of terrorist sounds}

The TNO automatic transient detector was adapted for real time detections on the streaming data with the four used hydrophones. A Graphical User Interface (GUI) was developed to display in real time the acoustical data available on the four available sensors.

For monitoring, the first step is the display of the recorded data as a "multi-channel LOw Frequency Analysis and Recording (LOFAR)" type of display: all four channels are presented at the same time in the frequency domain and updated real-time. An example of the output is shown in Figure 8.

The second step is automatic detection of the suspicious transients. This is achieved by the combined use of a power-law integrator and Page test detection algorithms, which act on all channels separately. The power-law integrator is robust against varying signal bandwidth while the Page test detector is an optimum detector for signals with an unknown duration as mentioned before. The power-law/Page test detector combination has proven to be very successful in detecting suspicious sounds. After preprocessing and normalisation of the raw acoustic data, the power-law/Page test detector is applied for 
automated detection and extraction of the signals. The normalisation is an adaptive process in which the background is continuously measured and averaged, and then subtracted from the data. In this way, only transient signals, which emerge in the stationary background are noticed. This step equalises the stationary background noise.

The detector processes two frequency bands simultaneously: The large frequency band from $1 \mathrm{~Hz}$ to 2 $\mathrm{kHz}$ is optimal for broad band sounds such as hammering and the lower frequency band from 0 to $500 \mathrm{~Hz}$ is optimal for the detection of pumps.

After the detection of a transient, the start and stop times of this transient are known and the transient can be stored. The stored transient files are displayed on the lower right part of the screen. This allows for further analysis and classification.

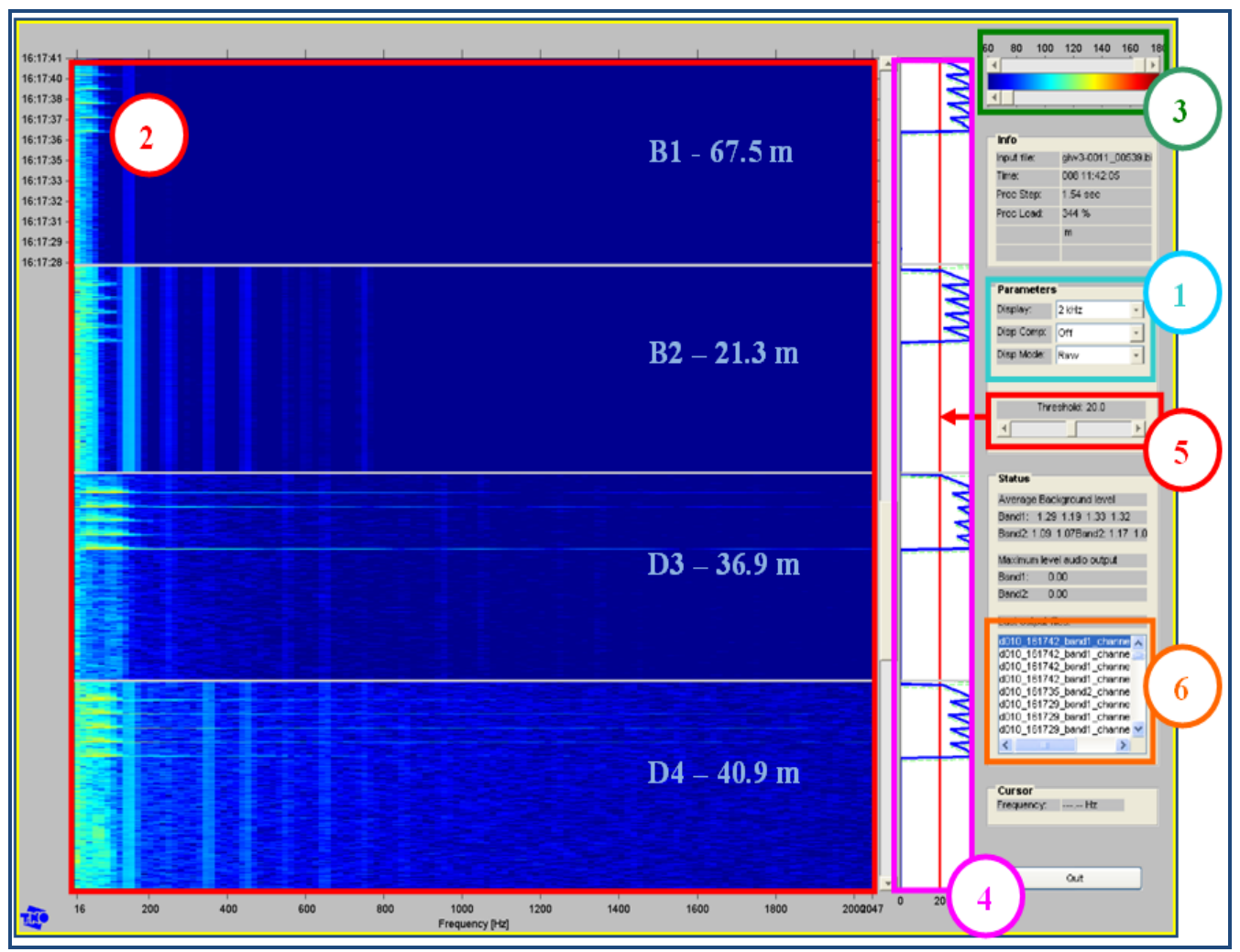

Figure 8 - Detector's GUI: example of strong hammer ticks with the big metal hammer on the injection point and records on the four sensor's channels. 1: Main display parameters including frequency band selection and normalization; 2: Multi-channel LOFAR display (colorbar can be adjusted in 3); 4: Page-test output (threshold can be adjusted in 5); 6: overview of last detected transient sound files.

\section{Classification of detected sounds}

Most classification techniques used in signal processing require accurate knowledge of the measurement environment, signals and false alarms. They are usually focused on limited and well defined types of signals and require a large signal database and careful tuning. Within the framework of this phase the measurement environment was new (new location, new acquisition and processing system) and the signals database was poor: some hammer ticks sounds were recorded in various measurement environments but there was hardly any data available on pumps (only one measurement) and false alarms. An essential step was therefore to gather enough data for development and training purposes. 
With these considerations in mind it was therefore decided to implement two types of classification techniques:

- Manual operator classification to build the database on the spot during the measurements using the operator hearing and visual skills and the known transient characteristics studied in previous phases. A quick look analysis tool was developed based on real time audio and spectrogram analysis (Figure 9).

- $\quad$ Automated classification based on spectrogram features analysis using image processing. This technique is inspired from previous work on classification of marine mammal sounds during which signals with similar structures (harmonic and click) were successfully classified (Van IJsselmuide, 2004 and Beerens, 2005). It was implemented prior to the experiments. For test and development, simulated data, based on available raw data from the previous phases (mostly hammer ticks) and on the new detector specifications, was used. Training efforts could only be performed post-trial once more data were available. It is the most delicate stage and very fine tuning of the classification features and thresholds is required before decent classification results on a whole experiment (including transients, false alarms and background noise) can be reached with limited interaction of the operator.

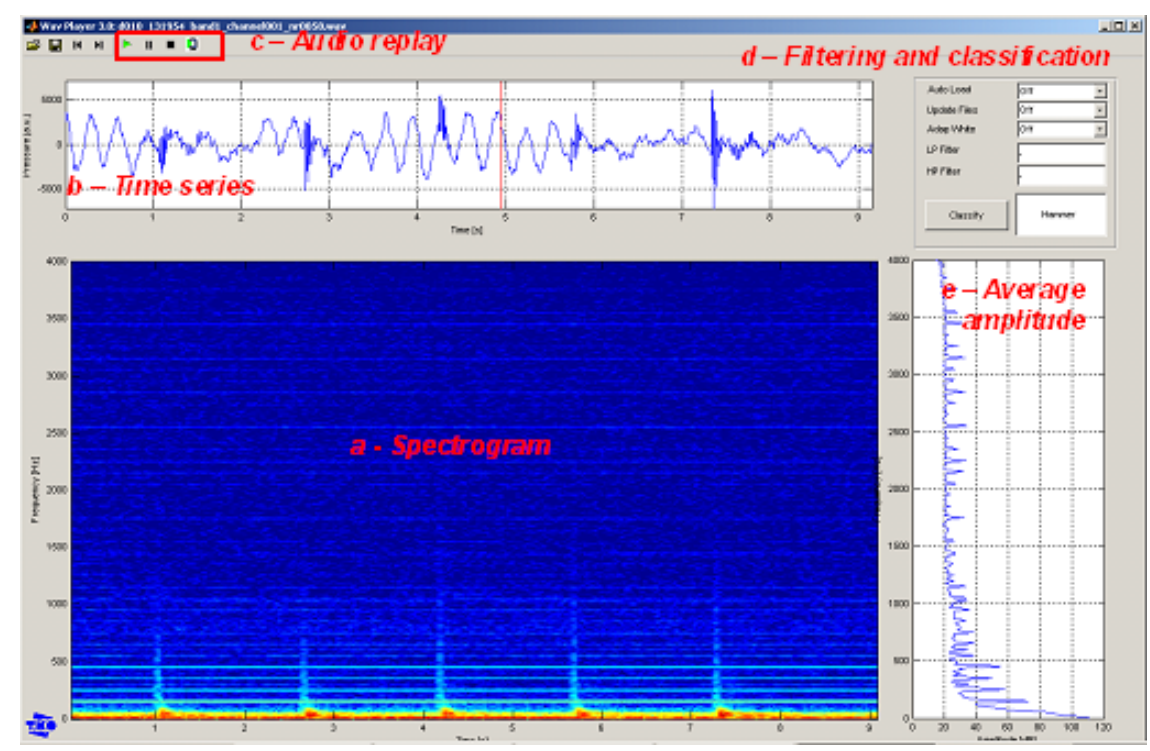

Figure 9 - Screen shot of manual classifier: audio and spectrogram analysis tool (example with 5 hammer ticks): a - spectrogram and $b$ - time series allow for visual analysis of the recorded transient; c- audio-player; d- filtering to improve visualization and access to classification; $e$ - average amplitude of the recorded signal in time.

\section{Results}

For time reasons, three experiments were preselected for in depth analysis. They were particularly interesting in order to test hammer tick series and pump sounds detection and classification with the building's pump on or off.

A first remark about the building's pump (full green circle in Figure 7, right hand side): since it is an extra and unsupervised electrical pump in the measurement network, it was a large disturbance for the measurements in particular for the hydrophone located on B1, which was the closest. When the pump is on, it saturates the detector on at least B1 and D3. After 15 seconds to 1 minute (could be slightly adjusted with the detector settings), the adaptive detection process assimilate the pump noise as a background noise. This is unfortunate since B1 was also the detection point at the largest distance from the injection point $(>60 \mathrm{~m})$. However, results when the building's pump was off indicate that missed detections resulted more from the tested pump itself rather than a larger distance from the injection point. 
Global results for detection on B1 located at $67.5 \mathrm{~m}$. from the injection point on the building waterpipe (38.5\% of events detected) confirm the previous remark. In particular, the results on the hammer ticks series (54\% performed while the building's pump was on) are in line with the qualitative analysis (only 17 $\%$ detection in total). Results with the manual pump are however very good. D3 located at $36.9 \mathrm{~m}$. from the injection point on the building waterpipe had the best results of all channels (75.5\%) for all types of events, and backround noise conditions. Results on D4 which is located only $4 \mathrm{~m}$. further from the injection point ( $40.9 \mathrm{~m}$ ) but outside of the building's network are much lower in comparison (58\%). B2 located at only $21.3 \mathrm{~m}$. from the injection point outside from the building's network performed slightly better than B1 (47.5\%) although one might have expected results considering the reduced distance.

Concerning false alarm rates, D3 (66.5\%) and D4 (66.5\%) have a much lower false alarm detection ratio than B1 (73.3\%) and B2 (74 \%) which come to confirm the qualitative observation on the real-time monitoring results that service tap hydrophones are more efficient than fire hydrants hydrophones. B2 which was the closest to the injection point $(21.3 \mathrm{~m})$ while it was pretty far from the building's pump has the highest false alarm rate which is consistent with the low event detection performance observed in the previous tables. This is probably the result of a complex propagation path as B2 was closest to the point were the building's water pipe connection and outside of the direct building water pipe network.

\section{CONCLUSION AND DISCUSSION}

During this project, a good comprehension of the acoustic monitoring of water pipes has been gathered and validated via experimental support: the absorption model for sounds in water pipes has been validated by the numerous datasets recorded in various conditions. The focus was set on PVC pipes of $110 \mathrm{~mm}$ diameter, which are widely used in the Netherlands. The detection and classification algorithms should not, in theory, be affected significantly by other diameters or materials, only the detection ranges would need to be experimentally tested.

The TNO monitoring system developed during this whole project is still an experimental system but it has proven its possibilities for protection of buildings against terrorist intrusion in the drinking water infrastructure in the context of our semi-realistic experiment. It can monitor and detect abnormal sounds on four channels real time and $90 \%$ of the 35 analysed events during the controlled test were detected at least once on one of the hydrophones of the measurement system.

More sounds can be incorporated to the detection/classification software suite developed. Most of the installation sounds (hammering, drilling, digging, mounting etc.) were detected up to $108 \mathrm{~m}$ during the last phase. Conversation sounds or speech sounds (swearing), originally not preselected as controlled tested transients, could also be detected at a distance up to $30 \mathrm{~m}$. Since the injection point (a service tap) is normally buried, if one digs a hole and talks next to the injection point this is considered as "characteristic" since otherwise it wouldn't be detected at all. These new sound types could be more extensively investigated and integrated in the classification algorithm, they will give an earlier warning of a possible terrorist attack. Furthermore, the talks could be heard real time by an operator, giving some insight on the terrorists' intentions.

\section{Recommendations}

The current system is an experimental design and a few limitations exist: A maximum number of four channels can currently be monitored simultaneously. In principle this number can be extended as widely as needed. Another current limitation is the maximum cable length available for the sensors: a fire hydrant's sensor cannot be placed further than $70 \mathrm{~m}$ and a service tap $110 \mathrm{~m}$ from the data acquisition 
system. Of course, these are more practical than physical limitations and in a final system, the use of longer cables or wireless solutions can be investigated as well. In order to help with the data analysis, the three main processing steps, after data acquisition, were performed on different PCs during the experiment: one for the raw data, one for the detection and one for the classification, all linked in a network to allow data exchange from one step to the other. A final version of the monitoring system could only use one PC (or laptop) for all these processing steps.

As mentioned in the introduction, leakage sounds can also be monitored with the developed acoustical monitoring system. However, more effort is needed to develop a specific and robust classification algorithm.

\section{ACKNOWLEDGMENT}

The authors would like to thank Paul van Walree, Pascal de Theije, Tilly Driesenaar, Christ de Jong, Joachim Golliard, Hugo Romijn, Wim Groen, Jeroen Janmaat and Adri Gerk from TNO for their precious help during the project. Hydron Midden-Nederland (Vitens) and Brabant Water are acknowledged for their field support during the experimental phases.

\section{REFERENCES}

Abraham, D.A. and Stahl, R.J. (1996), "Rapid detection of signals with unknown frequency content using Page’s test”, Proceedings of 1996 Conference on information Sciences and Systems, 809-814.

Beerens, S.P., Kromjongh, J., Spellen, M. van and IJsselmuide, S.P. van. (2005) "Sea trial of Delphinus; a towed array for marine mammal detection”, Proceedings of the European conference on Undersea Defence Technology (UDT), June 21-23, Amsterdam, The Netherlands.

Hiyoshi, Y., Tsuchiya, T., Naoi, Futa, J.K. and Kikuchi, T. (2004) "The difference in the low frequency sound propagation across the North Pacific sound communication in various species of Baleen Whales”, OCEANS '04. MTTS/IEEE TECHNO-OCEAN '04,Vol. 4, 2308-2315.

IJsselmuide, S.P. van and Beerens, S.P. (2004) "Detection and classification of marine mammals using an LFAS array”, Canadian Acoustics, 32(2).

Page E.S. (1954), “Continuous inspection schemes”, Biometrika, 41, 100-114.

Quesson, B.A.J., Sheldon-Robert, M.K., Groen, W.H.M., Driesenaar, M.L. (2008) - “Acoustic water pipe monitoring: Phase 3: Building protection”, TNO report, The Hague

Theije, P.A.M. de, Quesson, B.A.J., Golliard, J., Walree, P.A. van and Jong, C.A.F. de (2006) “Acoustic water pipe monitoring Phase II: Experimental support”, TNO-DV 2006-C364 (TNO report), The Hague

Walree, P.A. van, Beerens, S.P., Jong, C.A.F. de, Smeulers, J.P.M. and Alberts, G.J.N. (2004) “Acoustic water pipe monitoring Phase I: Knowledge creation”, FEL-04-C155 (TNO report), The Hague 